[5] S. X. Ding, T. Jeinsch, P. M. Frank, and E. L. Ding, "A unified approach to the optimization of fault detection systems," Int. J. Adaptive Control. Signal Processing, vol. 14, no. 7, pp. 725-745, 2000.

[6] S. X. Ding, M. Zhong, and B. Tang, "An LMI approach to the design of fault detection filter for time-delay LTI systems with unknown inputs," in Proc. Amer. Control Conf., Arlington, VA, 2001, pp. 2137-2142.

[7] B. Hassibi, A. H. Sayed, and K. T. , Indefinite Quadratic Estimation and Control: A Unified Approach to $\mathrm{H}_{2}$ and $\mathrm{H}_{\infty}$ Theories. Philadelphia, PA: SIAM, 1999.

[8] D. Henry and A. Zolghadri, "Norm-based design of robust FDI schemes for uncertain systems under feedback control: Comparison of two approaches," Control Eng. Practice, vol. 14, no. 6, pp. 1081-1097, 2006.

[9] X. Li and K. Zhou, "A time domain approach to robust fault detection of linear time-varying systems," Automatica, vol. 45, no. 1, pp. 94-102, 2009.

[10] N. Liu and K. Zhou, "Optimal robust fault detection for linear discrete time systems," in Proc. 46th IEEE Conf. Decis. Control, New Orleans, LA, 2007, pp. 989-994.

[11] G. Tadmor and L. Mirkin, " $H_{\infty}$ control and estimation with previewPart II: Fixed-size ARE solutions in discrete time," IEEE Trans. Autom. Control, vol. 50, no. 1, pp. 29-40, Jan. 2005.

[12] J. Wang, G. Yang, and J. Liu, "An LMI approach to $H_{-}$index and mixed $H_{-} / H_{\infty}$ fault detection observer design," Automatica, vol. 43, no. 9, pp. 1656-1665, 2007.

[13] H. Liu and G. Yang, "Fault detection observer design for linear discrete-time systems in finite frequency domain," in Proc. 46th IEEE Conf. Decision Control, New Orleans, LA, 2007, pp. 378-383.

[14] P. Zhang and S. X. Ding, "Observer-based fault detection of linear time-varying systems," (in German) Automatisierungstechniik, vol. 52, no. 8, pp. 370-376, 2004.

[15] P. Zhang, S. X. Ding, G. Wang, and D. Zhou, "Fault detection of linear discrete-time periodic systems," IEEE Trans. Autom. Control, vol. 50, no. 2, pp. 239-244, Feb. 2005.

[16] P. Zhang, S. X. Ding, G. Wang, and D. Zhou, "Disturbance decoupling in fault detection of linear periodic systems," Automatica, vol. 43, no. 8, pp. 1410-1417, 2007.

[17] H. Zhang, L. Xie, Y. C. Soh, and D. Zhang, " $H_{\infty}$ fixed-lag smoothing for discrete linear time-varying systems," Automatica, vol. 41, no. 5, pp. 839-846, 2005.

[18] H. Zhang and L. Xie, Control and Estimation of Systems With Input/ Output Delays. Berlin, Germany: Springer, 2007.

[19] M. Zhong, S. X. Ding, J. Lam, and H. Wang, "LMI approach to design robust fault detection filter for uncertain LTI systems," Automatica, vol. 39 , no. 3, pp. 543-550, 2003.

[20] M. Zhong, S. Liu, and H. Zhao, "Krein space-based $H_{\infty}$ fault estimation for linear discrete time-varying systems," ACTA Automatica Sinica, vol. 34, no. 12, pp. 1529-1533, 2008.

\section{Necessary and Sufficient Conditions for Analysis and Synthesis of Markov Jump Linear Systems With Incomplete Transition Descriptions}

\author{
Lixian Zhang and James Lam
}

\begin{abstract}
This technical note is concerned with exploring a new approach for the analysis and synthesis for Markov jump linear systems with incomplete transition descriptions. In the study, not all the elements of the transition rate matrices (TRMs) in continuous-time domain, or transition probability matrices (TPMs) in discrete-time domain are assumed to be known. By fully considering the properties of the TRMs and TPMs, and the convexity of the uncertain domains, necessary and sufficient criteria of stability and stabilization are obtained in both continuous and discrete time. Numerical examples are used to illustrate the results.
\end{abstract}

Index Terms-Markov jump linear systems, stability, stabilization.

\section{INTRODUCTION}

Markov jump linear system (MJLS) is a class of multi-modal systems in which the transitions among different modes are governed by a Markov chain. The studies of these systems are motivated by the powerful modeling capability of Markov chains in practical applications, and many useful results have been obtained, see [1]-[11] for instance. The concepts of semi-Markov chain, hidden Markov chain, time-nonhomogeneous Markov chain, have also been imported to the control community in recent years and have promoted many applications of MJLSs [12]-[14]. However, in most of the studies, complete knowledge of the mode transitions is required as a prerequisite for analysis and synthesis of MJLSs. This means that the transition probabilities (TPs) of the underlying Markov chain are assumed to be completely known. However, in practice, incomplete TPs are often encountered especially if adequate samples of the transitions are costly or time-consuming to obtain. Examples with such difficulties can be found in many fields, such as communication systems with delay variations and packet losses, biochemical systems with diverse changes of environmental conditions, temperature, humidity.

To relax the assumption that all the TPs are known, a new concept for MJLSs with partially unknown TPs is proposed [15] and a series of studies have been carried out [16]-[18]. The proposed systems are therefore more general, by which much more complex switching phenomena can be modeled. Meanwhile, as two extreme cases, the so-called switched systems under arbitrary switching [19], [20] and the conventional Markov jump systems are covered in the framework. However, although the works laid a conceptual foundation for analysis

Manuscript received December 01, 2009; revised February 03, 2010 and March 12, 2010 First published April 01, 2010; current version published July 08, 2010. This work was supported in part by RGC HKU 7137/09E, National Natural Science Foundation of China (60904001/F030107), Fund of Ministry of Education of China (20092302120071), China Postdoctoral Science Foundation (20090460069), Outstanding Youth Science Fund of China (60825303), 973 Project (2009CB320600) in China, Major program of National Natural Science Foundation of China (90916005/A0202), Heilongjiang Postdoctoral Science Foundation, China, and Overseas Talents Foundation of Harbin Institute of Technology. Recommended by Associated Editor P. Shi.

L. X. Zhang is with the Space Control and Inertial Technology Research Center, Harbin Institute of Technology, Harbin, Heilongjiang 150001, China (e-mail: lixianzhang@hit.edu.cn).

J. Lam is with the Department of Mechanical Engineering, University of Hong Kong, Hong Kong, China (e-mail: james.lam@hku.hk).

Digital Object Identifier 10.1109/TAC.2010.2046607 
and synthesis of MJLSs, the approach developed still has room for improvement in terms of conservatism. In fact, the properties of both the transition rate matrix (TRM) in continuous-time domain and the transition probability matrix (TPM) in discrete-time domain have not been fully used.

In this technical note, a new approach will be explored for the analysis and synthesis of MJLSs with incomplete description of their transitions. Using the properties that the sum of each row is zero in a TRM or one in a TPM, together with the convexity of the uncertain domains, necessary and sufficient conditions for the stability analysis and stabilization synthesis problems are first derived for both continuous-time and discrete-time cases. The conservatism in the previous studies is eliminated by considering the fact that the unknown elements of each row in TRM or TPM form a polytope. Moreover, for the continuous-time case, the difficulty that the unknown elements contain diagonal elements is also overcome by introducing a lower bound of the diagonal element without additional conservatism. The rest of the technical note is organized as follows. We formulate the considered systems in Section II. Section III is devoted to the issue of stability and stabilization for the system in both continuous-time and discrete-time cases. Numerical examples are provided to demonstrate the theoretical findings. The technical note is concluded in Section IV.

Notation: The notation used in this technical note is standard. The superscript " $T$ " stands for matrix transposition, $\mathbb{R}^{n}$ denotes the $n$ dimensional Euclidean space; $\mathbb{N}^{+}$represents the sets of positive integers, respectively. For the notation $(\Omega, \mathcal{F}, \mathcal{P}), \Omega$ represents the sample space, $\mathcal{F}$ is the $\sigma$-algebra of subsets of the sample space and $\mathcal{P}$ is the probability measure on $\mathcal{F}$. E[-] stands for the mathematical expectation. In addition, in symmetric block matrices or long matrix expressions, we use $*$ as an ellipsis for the terms that are introduced by symmetry and $\operatorname{diag}\left\{M_{1}, M_{2}, \ldots, M_{N}\right\}$ stands for a block-diagonal matrix constituted by $M_{1}, M_{2}, \ldots, M_{N}$. The notation $P>0(\geq 0)$ means $P$ is real symmetric positive (semi-positive) definite, and $M_{i}$ is adopted to denote $M(i)$ for brevity. $I$ and 0 represent respectively, identity matrix and zero matrix. Matrices, if their dimensions are not explicitly stated, are assumed to be compatible for algebraic operations.

\section{PRELIMINARIES}

Given the probability space $(\Omega, \mathcal{F}, \mathcal{P})$ and consider the following continuous-time and discrete-time MJLS, respectively:

$$
\dot{x}(t)=A\left(r_{t}\right) x(t)+B\left(r_{t}\right) u(t)
$$

and

$$
x(k+1)=A\left(r_{k}\right) x(k)+B\left(r_{k}\right) u(k)
$$

where $x(t) \in \mathbb{R}^{n}$ (respectively, $\left.x(k)\right)$ is the state vector and $u(t) \in \mathbb{R}^{l}$ (respectively, $u(k))$ is the control input. The stochastic process $\left\{r_{t}, t \geq\right.$ $0\}$ (respectively, the Markov chain $\left\{r_{k}, k \geq 0\right\}$ ), taking values in a finite set $\mathcal{I} \triangleq\{1, \ldots, N\}$, governs the switching among the different system modes. In the continuous-time MJLS, $\left\{r_{t}, t \geq 0\right\}$ is a continuous-time, discrete-state homogeneous Markov process and has the following mode transition probabilities:

$$
\operatorname{Pr}\left(r_{t+h}=j \mid r_{t}=i\right)= \begin{cases}\lambda_{i j} h+o(h), & \text { if } j \neq i \\ 1+\lambda_{i i} h+o(h), & \text { if } j=i\end{cases}
$$

where $h>0, \lim _{h \rightarrow 0}(o(h) / h)=0$ and $\lambda_{i j} \geq 0(i, j \in \mathcal{I}, j \neq i)$ denotes the switching rate from mode $i$ at time $t$ to mode $j$ at time $t+h$, and $\lambda_{i i}=-\sum_{j=1, j \neq i} \lambda_{i j}$ for all $i \in \mathcal{I}$. Hence, the transition rate matrix (TRM) in the Markov process is given by

$$
\boldsymbol{\Lambda}=\left[\begin{array}{cccc}
\lambda_{11} & \lambda_{12} & \cdots & \lambda_{1 N} \\
\lambda_{21} & \lambda_{22} & \cdots & \lambda_{2 N} \\
& & \ddots & \\
\lambda_{N 1} & \lambda_{N 2} & \cdots & \lambda_{N N}
\end{array}\right]
$$

For the discrete-time case, the process $\left\{r_{k}, k \geq 0\right\}$ is described by a discrete-time homogeneous Markov chain, which takes values in finite set $\mathcal{I}$ with mode transition probabilities

$$
\operatorname{Pr}\left(r_{k+1}=j \mid r_{k}=i\right)=\pi_{i j}
$$

where $\pi_{i j} \geq 0, \forall i, j \in \mathcal{I}$, and $\sum_{j=1}^{N} \pi_{i j}=1$. Likewise, the transition probability matrix (TPM) is given by

$$
\boldsymbol{\Pi}=\left[\begin{array}{cccc}
\pi_{11} & \pi_{12} & \cdots & \pi_{1 N} \\
\pi_{21} & \pi_{22} & \cdots & \pi_{2 N} \\
& & \ddots & \\
\pi_{N 1} & \pi_{N 2} & \cdots & \pi_{N N}
\end{array}\right]
$$

The set $\mathcal{I}$ contains $N$ modes of system (1) (or system (2)) and for $r_{t}=$ $i \in \mathcal{I}$ (respectively, $r_{k}=i$ ), the system matrices of the $i^{t h}$ mode are denoted by $A_{i}, B_{i}, C_{i}, D_{i}, E_{i}, F_{i}$, which are real and known.

The transition rates or probabilities described above are considered to be partially available, that is, some elements in matrix $\boldsymbol{\Lambda}$ or $\boldsymbol{\Pi}$ are unknown. Take system (1) or system (2) with 4 operation modes for example, the TRM $\Lambda$ or TPM $\boldsymbol{\Pi}$ may be written as

$$
\left[\begin{array}{llll}
\lambda_{11} & \hat{\lambda}_{12} & \lambda_{13} & \hat{\lambda}_{14} \\
\hat{\lambda}_{21} & \hat{\lambda}_{22} & \hat{\lambda}_{23} & \lambda_{24} \\
\hat{\lambda}_{31} & \lambda_{32} & \lambda_{33} & \hat{\lambda}_{34} \\
\hat{\lambda}_{41} & \hat{\lambda}_{42} & \lambda_{43} & \lambda_{44}
\end{array}\right],\left[\begin{array}{llll}
\pi_{11} & \hat{\pi}_{12} & \pi_{13} & \hat{\pi}_{14} \\
\hat{\pi}_{21} & \hat{\pi}_{22} & \hat{\pi}_{23} & \pi_{24} \\
\pi_{31} & \hat{\pi}_{32} & \pi_{33} & \hat{\pi}_{34} \\
\hat{\pi}_{41} & \hat{\pi}_{42} & \pi_{43} & \pi_{44}
\end{array}\right]
$$

where each unknown element is labeled with a hat "."

For convenience, $\forall i \in \mathcal{I}$, we denote

$$
\begin{aligned}
& \mathcal{I}_{\mathcal{K}}^{(i)} \triangleq\left\{j: \lambda_{i j}\left(\text { or } \pi_{i j}\right) \text { is known }\right\} \\
& \mathcal{I}_{\mathcal{U} \mathcal{K}}^{(i)} \triangleq\left\{j: \lambda_{i j}\left(\text { or } \pi_{i j}\right) \text { is unknown }\right\}
\end{aligned}
$$

In addition, if $\mathcal{I}_{\mathcal{K}}^{(i)} \neq \emptyset, \mathcal{I}_{\mathcal{K}}^{(i)}$ is further described as

$$
\mathcal{I}_{\mathcal{K}}^{(i)}=\left\{\mathcal{K}_{1}, \mathcal{K}_{2}, \ldots, \mathcal{K}_{m_{i}}\right\}, \quad m_{i} \in\{1,2, \ldots, N-2\}
$$

where $\mathcal{K}_{s} \in \mathbb{N}^{+}, s \in\left\{1,2, \ldots, m_{i}\right\}$, represents the index of the $s^{t h}$ known element in the $i^{\text {th }}$ row of matrix $\Lambda$ or $\boldsymbol{\Pi}$. Also, throughout the technical note, we denote

$$
\lambda_{\mathcal{K}}^{(i)} \triangleq \sum_{j \in \mathcal{I}_{\mathcal{K}}^{(i)}} \lambda_{i j}, \quad \pi_{\mathcal{K}}^{(i)} \triangleq \sum_{j \in \mathcal{I}_{\mathcal{K}}^{(i)}} \pi_{i j} .
$$

In the continuous-time case, when $\hat{\lambda}_{i i}$ is unknown, it is necessary to provide a lower bound $\lambda_{d}^{(i)}$ for it and we have $\lambda_{d}^{(i)} \leq-\lambda_{\mathcal{K}}^{(i)}$.

Remark 1: The case $m_{i}=N-1, \forall i \in \mathcal{I}$, is excluded in (4), which means if we have only one unknown element, one can naturally calculate it from the known elements in each row and the TRM or TPM property.

For MJLSs, the following stability definition will be used [1], [2].

Definition 1: System (1) (respectively, (2)) is said to be stochastically stable if for $u(t) \equiv 0$ (respectively, $u(k) \equiv 0$ ) and every initial condition $x_{0} \in \mathbb{R}^{n}$ and $r_{0} \in \mathcal{I}$, the following holds:

$$
\begin{gathered}
E\left\{\int_{0}^{\infty}\|x(t)\|^{2} \mid x_{0}, r_{0}\right\}<\infty \\
\text { (respectively, } \left.E\left\{\sum_{k=0}^{\infty}\|x(k)\|^{2} \mid x_{0}, r_{0}\right\}<\infty\right) .
\end{gathered}
$$




\section{STABILITY AND STABILIZATION}

In this section, we will derive the stochastic stability criteria for system (1) and system (2) when the transition rates or probabilities are partially unknown, and to design a state-feedback stabilizing controller such that the closed-loop system is stochastically stable. The mode-dependent controller considered here has the form

$$
u(t)=K\left(r_{t}\right) x(t) \quad\left(\text { respectively }, \quad u(k)=K\left(r_{k}\right) x(k)\right)
$$

where $K_{i}\left(\forall r_{t}=i \in \mathcal{I}\right.$, or $\left.r_{k}=i \in \mathcal{I}\right)$ are the controller gains to be determined. First, we provide the following preliminary stability results for MJLSs with completely known TRM or TPM.

Lemma 1 ([1]): System (1) (with $u(t) \equiv 0$ ) is stochastically stable if and only if there exists a set of positive-definite matrices $P_{i}, i \in \mathcal{I}$, satisfying

$$
A_{i}^{T} P_{i}+P_{i} A_{i}+\mathcal{P}^{(i)}<0
$$

where $\mathcal{P}^{(i)} \triangleq \sum_{j \in \mathcal{I}} \lambda_{i j} P_{j}$.

Lemma 2 ([2]): System (2) (with $u(k) \equiv 0$ ) is stochastically stable if and only if there exists a set of positive-definite matrices $P_{i}, i \in \mathcal{I}$, satisfying

$$
A_{i}^{T} \mathcal{P}^{(i)} A_{i}-P_{i}<0
$$

where $\mathcal{P}^{(i)} \triangleq \sum_{j \in \mathcal{I}} \pi_{i j} P_{j}$.

\section{A. Continuous-Time Case}

Let us first give the stability result for the unforced system (1) (with $u(t) \equiv 0)$. The following theorem presents a necessary and sufficient condition on the stochastic stability of the considered system with partially unknown transition rates.

Theorem 1: Consider unforced system (1) with partially unknown transition rates. The corresponding system is stochastically stable if and only if there exists a set of matrices $P_{i}>0, i \in \mathcal{I}$, such that, $\forall i \in \mathcal{I}$

$$
\begin{aligned}
& A_{i}^{T} P_{i}+P_{i} A_{i}+\mathcal{P}_{\mathcal{K}}^{(i)}-\lambda_{\mathcal{K}}^{(i)} P_{j}<0, \\
& \quad \forall j \in \mathcal{I}_{\mathcal{U} \mathcal{K}}^{(i)}, \quad \text { if } i \in \mathcal{I}_{\mathcal{K}}^{(i)} \\
& A_{i}^{T} P_{i}+P_{i} A_{i}+\mathcal{P}_{\mathcal{K}}^{(i)}+\lambda_{d}^{(i)} P_{i}-\lambda_{d}^{(i)} P_{j}-\lambda_{\mathcal{K}}^{(i)} P_{j}<0, \\
& \quad \forall j \in \mathcal{I}_{\mathcal{U} \mathcal{K}}^{(i)}, \quad \text { if } i \in \mathcal{I}_{\mathcal{U} \mathcal{K}}^{(i)}
\end{aligned}
$$

where $\mathcal{P}_{\mathcal{K}}^{(i)} \triangleq \sum_{j \in \mathcal{I}_{\mathcal{K}}^{(i)}} \lambda_{i j} P_{j}$ and $\lambda_{d}^{(i)}$ is a given lower bound for the unknown diagonal element.

Proof: We shall separate the proof into two cases, $i \in \mathcal{I}_{\mathcal{K}}^{(i)}$ and $i \in \mathcal{I}_{\mathcal{U K}}^{(i)}$, and bear in mind that system (1) is stochastically stable if and only if (6) holds.

1) Case 1: $i \in \mathcal{I}_{\mathcal{K}}^{(i)}$.

It should be first noted that in this case one has $\lambda_{\mathcal{K}}^{(i)} \leq 0$. We only need to consider $\lambda_{\mathcal{K}}^{(i)}<0$ here since $\lambda_{\mathcal{K}}^{(i)}=0$ means the elements in the $i^{t h}$ row of the TRM are known.

Now we rewrite the left-hand side of (6) as

$$
\begin{aligned}
\Theta_{i} & \triangleq A_{i}^{T} P_{i}+P_{i} A_{i}+\mathcal{P}_{\mathcal{K}}^{(i)}+\sum_{j \in \mathcal{I}_{\mathcal{U} \mathcal{K}}^{(i)}} \hat{\lambda}_{i j} P_{j} \\
& =A_{i}^{T} P_{i}+P_{i} A_{i}+\mathcal{P}_{\mathcal{K}}^{(i)}-\lambda_{\mathcal{K}}^{(i)} \sum_{j \in \mathcal{I}_{\mathcal{U} \mathcal{K}}^{(i)}} \frac{\hat{\lambda}_{i j}}{-\lambda_{\mathcal{K}}^{(i)}} P_{j}
\end{aligned}
$$

where the elements $\hat{\lambda}_{i j}, \forall j \in \mathcal{I}_{\mathcal{U} \mathcal{K}}^{(i)}$, are unknown. Since we have $0 \leq\left(\hat{\lambda}_{i j} /-\lambda_{\mathcal{K}}^{(i)}\right) \leq 1$ and $\sum_{j \in \mathcal{I}_{\mathcal{U} \mathcal{K}}^{(i)}}\left(\hat{\lambda}_{i j} /-\lambda_{\mathcal{K}}^{(i)}\right)=1$, we know that

$$
\Theta_{i}=\sum_{j \in \mathcal{I}_{\mathcal{U} \mathcal{K}}^{(i)}} \frac{\hat{\lambda}_{i j}}{-\lambda_{\mathcal{K}}^{(i)}}\left[A_{i}^{T} P_{i}+P_{i} A_{i}+\mathcal{P}_{\mathcal{K}}^{i}-\lambda_{\mathcal{K}}^{(i)} P_{j}\right]
$$

Therefore, for $0 \leq \hat{\lambda}_{i j} \leq-\lambda_{\mathcal{K}}^{(i)}, \Theta_{i}<0$ is equivalent to $A_{i}^{T} P_{i}+$ $P_{i} A_{i}+\mathcal{P}_{\mathcal{K}}^{i}-\lambda_{\mathcal{K}}^{(i)} P_{j}<0, \forall j \in \mathcal{I}_{\mathcal{U} \mathcal{K}}^{(i)}$, which implies that, in the presence of unknown elements $\hat{\lambda}_{i j}$, the system stability is ensured if and only if (8) holds.

2) Case 2: $i \in \mathcal{I}_{\mathcal{U} \mathcal{K}}^{(i)}$.

In this case, $\hat{\lambda}_{i i}$ is unknown, $\lambda_{\mathcal{K}}^{(i)} \geq 0$ and $\hat{\lambda}_{i i} \leq-\lambda_{\mathcal{K}}^{(i)}$. Also, we only consider $\hat{\lambda}_{i i}<-\lambda_{\mathcal{K}}^{(i)}$ here since if $\hat{\lambda}_{i i}=-\lambda_{\mathcal{K}}^{(i)}$, then the $i^{t h}$ row of the TRM is completely known.

Now the left-hand side of the stability condition in (6) can be rewritten as

$$
\begin{aligned}
\Theta_{i}= & A_{i}^{T} P_{i}+P_{i} A_{i}+\mathcal{P}_{\mathcal{K}}^{(i)}+\hat{\lambda}_{i i} P_{i}+\sum_{j \in \mathcal{I}_{\mathcal{U} \mathcal{K}}^{(i)}, j \neq i} \hat{\lambda}_{i j} P_{j} \\
= & A_{i}^{T} P_{i}+P_{i} A_{i}+\mathcal{P}_{\mathcal{K}}^{(i)}+\hat{\lambda}_{i i} P_{i} \\
& +\left(-\hat{\lambda}_{i i}-\lambda_{\mathcal{K}}^{(i)}\right) \sum_{j \in \mathcal{I}_{\mathcal{U} \mathcal{K}}^{(i)}, j \neq i} \frac{\hat{\lambda}_{i j}}{-\hat{\lambda}_{i i}-\lambda_{\mathcal{K}}^{(i)}} P_{j} .
\end{aligned}
$$

Likewise, since we have $0 \leq\left(\hat{\lambda}_{i j} /-\hat{\lambda}_{i i}-\lambda_{\mathcal{K}}^{(i)}\right) \leq 1$ and $\sum_{j \in \mathcal{I}_{\mathcal{U K}}^{(i)}, j \neq i}\left(\hat{\lambda}_{i j} /-\hat{\lambda}_{i i}-\lambda_{\mathcal{K}}^{(i)}\right)=1$, we know that

$$
\begin{aligned}
\Theta_{i}=\sum_{j \in \mathcal{I}_{\mathcal{U} \mathcal{K}}^{(i)}, j \neq i} \frac{\hat{\lambda}_{i j}}{-\hat{\lambda}_{i i}-\lambda_{\mathcal{K}}^{(i)}}\left[A_{i}^{T} P_{i}+P_{i} A_{i}+\mathcal{P}_{\mathcal{K}}^{(i)}\right. & \\
& \left.+\hat{\lambda}_{i i} P_{i}-\hat{\lambda}_{i i} P_{j}-\lambda_{\mathcal{K}}^{(i)} P_{j}\right]
\end{aligned}
$$

which means that $\Theta_{i}<0$ is equivalent to $\forall j \in \mathcal{I}_{\mathcal{U} \mathcal{K}}^{(i)}, j \neq i$

$$
A_{i}^{T} P_{i}+P_{i} A_{i}+\mathcal{P}_{\mathcal{K}}^{(i)}+\hat{\lambda}_{i i} P_{i}-\hat{\lambda}_{i i} P_{j}-\lambda_{\mathcal{K}}^{(i)} P_{j}<0 .
$$

As $\hat{\lambda}_{i i}$ is lower bounded by $\lambda_{d}^{(i)}$, we have

$$
\lambda_{d}^{(i)} \leq \hat{\lambda}_{i i}<-\lambda_{\mathcal{K}}^{(i)}
$$

which implies that $\hat{\lambda}_{i i}$ may take any value between $\left[\lambda_{d}^{(i)},-\lambda_{\mathcal{K}}^{(i)}+\right.$ $\epsilon]$ for some $\epsilon<0$ arbitrarily small. Then $\hat{\lambda}_{i i}$ can be further written as a convex combination

$$
\hat{\lambda}_{i i}=-\alpha \lambda_{\mathcal{K}}^{(i)}+\alpha \epsilon+(1-\alpha) \lambda_{d}^{(i)}
$$

where $\alpha$ takes value arbitrarily in [0,1]. Thus, (10) holds if and only if $\forall j \in \mathcal{I}_{\mathcal{U} \mathcal{K}}^{(i)}, j \neq i$

$A_{i}^{T} P_{i}+P_{i} A_{i}+\mathcal{P}_{\mathcal{K}}^{(i)}-\lambda_{\mathcal{K}}^{(i)} P_{i}+\lambda_{\mathcal{K}}^{(i)} P_{j}-\lambda_{\mathcal{K}}^{(i)} P_{j}+\epsilon\left(P_{i}-P_{j}\right)<0$

and

$A_{i}^{T} P_{i}+P_{i} A_{i}+\mathcal{P}_{\mathcal{K}}^{(i)}+\lambda_{d}^{(i)} P_{i}-\lambda_{d}^{(i)} P_{j}-\lambda_{\mathcal{K}}^{(i)} P_{j}<0$

simultaneously hold. Since $\epsilon$ is arbitrarily small, (11) holds if and only if

$$
A_{i}^{T} P_{i}+P_{i} A_{i}+\mathcal{P}_{\mathcal{K}}^{(i)}-\lambda_{\mathcal{K}}^{(i)} P_{i}<0
$$

which is the case in (12) when $j=i, \forall j \in \mathcal{I}_{\mathcal{U} \mathcal{K}}^{(i)}$. Hence (10) is equivalent to (9). 
Therefore, in the presence of unknown elements in the TRM, one can readily conclude that the system is stable if and only if (8) and (9) hold for $i \in \mathcal{I}_{\mathcal{K}}^{(i)}$ and $i \in \mathcal{I}_{\mathcal{U} \mathcal{K}}^{(i)}$, respectively.

Remark 2: The stability criterion developed in Theorem 1 is less conservative than the one obtained in [15]. More specifically, in Theorem 1 of [15], if $i \in \mathcal{I}_{\mathcal{K}}^{(i)}$, the conditions are

$$
\left\{\begin{array}{l}
\left(1+\lambda_{\mathcal{K}}^{(i)}\right)\left(A_{i}^{T} P_{i}+P_{i} A_{i}\right)+\mathcal{P}_{\mathcal{K}}^{(i)}<0 \\
A_{i}^{T} P_{i}+P_{i} A_{i}+P_{j} \leq 0
\end{array}\right.
$$

which, since $\lambda_{\mathcal{K}}^{(i)}<0$, ensure

$$
\left(1+\lambda_{\mathcal{K}}^{(i)}\right)\left(A_{i}^{T} P_{i}+P_{i} A_{i}\right)+\mathcal{P}_{\mathcal{K}}^{(i)}-\lambda_{\mathcal{K}}^{(i)}\left(A_{i}^{T} P_{i}+P_{i} A_{i}+P_{j}\right)<0
$$

which is (8). Also, if $i \in \mathcal{I}_{\mathcal{U} \mathcal{K}}^{(i)}$, the criteria in Theorem 1 of [15] are

$$
\begin{cases}\left(1+\lambda_{\mathcal{K}}^{(i)}\right)\left(A_{i}^{T} P_{i}+P_{i} A_{i}\right)+\mathcal{P}_{\mathcal{K}}^{(i)}<0 & \\ A_{i}^{T} P_{i}+P_{i} A_{i}+P_{j} \geq 0, \quad \forall j \in \mathcal{I}_{\mathcal{U} \mathcal{K}}^{(i)}, & j=i \\ A_{i}^{T} P_{i}+P_{i} A_{i}+P_{j} \leq 0, \quad \forall j \in \mathcal{I}_{\mathcal{U} \mathcal{K}}^{(i)}, & j \neq i .\end{cases}
$$

In this case, since $\lambda_{d}^{(i)}<0$ and $-\lambda_{d}^{(i)}-\lambda_{\mathcal{K}}^{(i)}>0$, we have

$$
\begin{aligned}
\left(1+\lambda_{\mathcal{K}}^{(i)}\right)\left(A_{i}^{T} P_{i}+P_{i} A_{i}\right)+ & \mathcal{P}_{\mathcal{K}}^{(i)}+\lambda_{d}^{(i)}\left(A_{i}^{T} P_{i}+P_{i} A_{i}+P_{i}\right)+ \\
& \left(-\lambda_{d}^{(i)}-\lambda_{\mathcal{K}}^{(i)}\right)\left(A_{i}^{T} P_{i}+P_{i} A_{i}+P_{j}\right)<0
\end{aligned}
$$

which guarantees

$A_{i}^{T} P_{i}+P_{i} A_{i}+\mathcal{P}_{\mathcal{K}}^{(i)}+\lambda_{d}^{(i)} P_{i}-\lambda_{d}^{(i)} P_{j}-\lambda_{\mathcal{K}}^{(i)} P_{j}<0, \quad \forall j \in \mathcal{I}_{\mathcal{U} \mathcal{K}}^{(i)}$

Therefore, the conditions (8), (9) are less conservative than (13). Note that the obtained conditions are without loss of generality since the lower bound, $\lambda_{d}^{(i)}$, of $\hat{\lambda}_{i i}$ is allowed to be arbitrarily negative.

Now let us consider the stabilization problem of system (1) in the presence of unknown elements in the TRM. The following theorem presents a necessary and sufficient criterion for the existence of a modedependent stabilizing controller of the form in (5).

Theorem 2: Consider system (1) with partially unknown transition rates. If there exist matrices $X_{i}>0$ and $Y_{i}, \forall i \in \mathcal{I}$ such that

$$
\begin{aligned}
& {\left[\begin{array}{ccc}
\Lambda_{i}+\lambda_{i i} X_{i} & \mathcal{T}_{\mathcal{K}}^{(i)} & \sqrt{-\lambda_{\mathcal{K}}^{(i)}} X_{i} \\
* & -\mathcal{X}_{\mathcal{K}}^{(i)} & 0 \\
* & * & -X_{j}
\end{array}\right]<0,} \\
& \forall j \in \mathcal{I}_{\mathcal{U} \mathcal{K}}^{(i)}, \quad \text { if } i \in \mathcal{I}_{\mathcal{K}}^{(i)} \\
& {\left[\begin{array}{ccc}
\Lambda_{i}+\lambda_{d}^{(i)} X_{i} & \mathcal{T}_{\mathcal{K}}^{(i)} & \sqrt{-\lambda_{d}^{(i)}-\lambda_{\mathcal{K}}^{(i)}} X_{i} \\
* & -\mathcal{X}_{\mathcal{K}}^{(i)} & 0 \\
* & * & -X_{j}
\end{array}\right]<0,} \\
& \forall j \in \mathcal{I}_{\mathcal{U} \mathcal{K}}^{(i)}, \quad \text { if } i \in \mathcal{I}_{\mathcal{U} \mathcal{K}}^{(i)}
\end{aligned}
$$

where $\Lambda_{i} \triangleq A_{i} X_{i}+X_{i} A_{i}^{T}+B_{i} Y_{i}+Y_{i}^{T} B_{i}^{T}$ and

$$
\begin{aligned}
& \mathcal{X}_{\mathcal{K}}^{(i)} \triangleq \operatorname{diag}\left[X_{\mathcal{K}_{1}}, \ldots, X_{\mathcal{K}_{m_{i}}}\right], \\
& \mathcal{T}_{\mathcal{K}}^{(i)} \triangleq\left[\sqrt{\lambda_{i \mathcal{K}_{1}}} X_{i}, \ldots, \sqrt{\lambda_{i \mathcal{K}_{m_{i}}}} X_{i}\right]
\end{aligned}
$$

and $\forall s \in\left\{1,2, \ldots, m_{i}\right\}, \mathcal{K}_{s}$ is described in (4), $\mathcal{K}_{s} \neq i$, then there exists a mode-dependent stabilizing controller of the form in (5) such that the closed-loop system is stochastically stable. Moreover, if the LMIs in (14), (15) have a solution, an admissible controller gain is given by

$$
K_{i}=Y_{i} X_{i}^{-1}
$$

Proof: Consider system (1) with the control input (5) and replace $A_{i}$ by $A_{i}+B_{i} K_{i}$ in (8), (9), respectively. Then, if $i \in \mathcal{I}_{\mathcal{K}}^{(i)}$, performing a congruence transformation to (8) by $P_{i}^{-1}$, we can obtain

$$
\begin{aligned}
&\left(A_{i}+B_{i} K_{i}\right) P_{i}^{-1}+P_{i}^{-1}\left(A_{i}+B_{i} K_{i}\right)^{T}+P_{i}^{-1} \mathcal{P}_{\mathcal{K}}^{(i)} P_{i}^{-1}- \\
& P_{i}^{-1} \lambda_{\mathcal{K}}^{(i)} P_{j} P_{i}^{-1}<0 .
\end{aligned}
$$

Setting $X_{i} \triangleq P_{i}^{-1}, Y_{i} \triangleq K_{i} X_{i}$ and considering (16), by Schur complement, one can obtain that (18) is equivalent to (14). In a similar way, if $i \in \mathcal{I}_{\mathcal{U} K}^{(i)},(15)$ can be worked out from (9). Meanwhile, due to $Y_{i}=K_{i} X_{i}$, the desired controller gain is given by (17).

Remark 3: It is noted from (15) that if the diagonal elements in the TRM contain unknown ones, the system stability, the existence of the admissible controller and the controller gains solution will be dependent on $\lambda_{d}^{(i)}$. This dependency, therefore, will reduce the conservatism existed in the previous " $\lambda_{d}^{(i)}$-independent" results obtained in [15].

\section{B. Discrete-Time Case}

The following theorem presents a necessary and sufficient condition on the stochastic stability of the unforced system (2) with partially unknown transition probabilities.

Theorem 3: Consider the unforced system (2) with partially unknown transition probabilities. The corresponding system is stochastically stable if and only if there exists a set of matrices $P_{i}>0, i \in \mathcal{I}$ such that

$$
A_{i}^{T}\left(\mathcal{P}_{\mathcal{K}}^{(i)}+\left(1-\pi_{\mathcal{K}}^{(i)}\right) P_{j}\right) A_{i}-P_{i}<0, \quad \forall j \in \mathcal{I}_{\mathcal{K}}^{(i)}
$$

where $\mathcal{P}_{\mathcal{K}}^{(i)} \triangleq \sum_{j \in \mathcal{I}_{\mathcal{K}}^{(i)}} \pi_{i j} P_{j}$.

Proof: It should be first noted that $\pi_{\mathcal{K}}^{(i)} \leq 1$ in the discrete-time case, and we exclude $\pi_{\mathcal{K}}^{(i)}=1$ here since it means that all the elements in the $i^{\text {th }}$ row are known.

Now the left-hand side of stability condition (7) in Lemma 2 can be rewritten as

$$
\begin{aligned}
\Psi_{i} & \triangleq A_{i}^{T}\left(\mathcal{P}_{\mathcal{K}}^{i}+\sum_{j \in \mathcal{I}_{\mathcal{U} \mathcal{K}}^{(i)}} \hat{\pi}_{i j} P_{j}\right) A_{i}-P_{i} \\
& =A_{i}^{T}\left(\mathcal{P}_{\mathcal{K}}^{i}+\left(1-\pi_{\mathcal{K}}^{(i)}\right) \sum_{j \in \mathcal{I}_{\mathcal{U} \mathcal{K}}^{(i)}} \frac{\hat{\pi}_{i j}}{1-\pi_{\mathcal{K}}^{(i)}} P_{j}\right) A_{i}-P_{i}
\end{aligned}
$$

where the elements $\hat{\pi}_{i j}, j \in \mathcal{I}_{\mathcal{U} \mathcal{K}}^{(i)}$, are unknown. Since $0 \leq\left(\hat{\pi}_{i j} / 1-\right.$ $\left.\pi_{\mathcal{K}}^{(i)}\right) \leq 1, \forall j \in \mathcal{I}_{\mathcal{U} \mathcal{K}}^{(i)}$ and $\sum_{j \in \mathcal{I}_{\mathcal{U} \mathcal{K}}^{(i)}}\left(\hat{\pi}_{i j} / 1-\pi_{\mathcal{K}}^{(i)}\right)=1$, we know that

$$
\Psi_{i}=\sum_{j \in \mathcal{I}_{\mathcal{U} \mathcal{K}}^{(i)}} \frac{\hat{\pi}_{i j}}{1-\pi_{\mathcal{K}}^{(i)}}\left[A_{i}^{T}\left(\mathcal{P}_{\mathcal{K}}^{(i)}+\left(1-\pi_{\mathcal{K}}^{(i)}\right) P_{j}\right) A_{i}-P_{i}\right]
$$

Therefore, for $0 \leq \hat{\pi}_{i j} \leq 1-\pi_{\mathcal{K}}^{(i)}, \Psi_{i}<0$ is equivalent to $A_{i}^{T}\left(\mathcal{P}_{\mathcal{K}}^{(i)}+\right.$ $\left.\left(1-\pi_{\mathcal{K}}^{(i)}\right) P_{j}\right) A_{i}-P_{i}<0, \forall j \in \mathcal{I}_{\mathcal{U} \mathcal{K}}^{(i)}$, which implies that, in the presence of unknown elements $\hat{\pi}_{i j}$, the system stability is ensured if and only if (19) holds.

Remark 4: Analogous to Remark 2 for the continuous-time case, the necessary and sufficient criterion developed in Theorem 3 is also less conservative when compared with Theorem 3 in [15], where the stability conditions are given by

$$
\begin{aligned}
A_{i}^{T} \mathcal{P}_{\mathcal{K}}^{(i)} A_{i}-\pi_{\mathcal{K}}^{(i)} P_{i} & <0 \\
A_{i}^{T} P_{j} A_{i}-P_{i} & <0, \quad \forall j \in \mathcal{I}_{\mathcal{U} \mathcal{K}}^{(i)} .
\end{aligned}
$$


The inequalities yield

$$
A_{i}^{T} \mathcal{P}_{\mathcal{K}}^{(i)} A_{i}-\pi_{\mathcal{K}}^{(i)} P_{i}+\left(1-\pi_{\mathcal{K}}^{(i)}\right)\left(A_{i}^{T} P_{j} A_{i}-P_{i}\right)<0, \quad \forall j \in \mathcal{I}_{\mathcal{U} \mathcal{K}}^{(i)}
$$

which is (19). Therefore, combined with Remark 1, it is seen that the approach adopted in Theorems 1 and 3 in this technical note, which uses the TRM or TPM property (the sum of all the elements in each row is zero or one), gives the necessary and sufficient criteria and are less conservative than the existing results.

Now consider the system (2) with control input $u(k)$, the following theorem presents a condition for the existence of a mode-dependent stabilizing controller with the form in (5).

Theorem 4: Consider system (2) with partially unknown transition probabilities. If there exist matrices $X_{i}>0$ and $Y_{i}, \forall i \in \mathcal{I}$ such that

$$
\left[\begin{array}{cc}
-X_{i} & {\left[\mathcal{L}_{\mathcal{K}}^{(i)}\left(A_{i} X_{i}+B_{i} Y_{i}\right)\right]^{T}} \\
* & -\mathcal{X}_{\mathcal{K}}^{(i)}
\end{array}\right]<0
$$

where

$$
\begin{aligned}
& \mathcal{L}_{\mathcal{K}}^{(i)} \triangleq\left[\sqrt{\pi_{i \mathcal{K}_{1}}} I, \ldots, \sqrt{\pi_{i \mathcal{K}_{m_{i}}}} I, \sqrt{1-\pi_{\mathcal{K}}^{(i)}} I\right]^{T} \\
& \mathcal{X}_{\mathcal{K}}^{(i)} \triangleq \operatorname{diag}\left[X_{\mathcal{K}_{1}}, \ldots, X_{\mathcal{K}_{m_{i}}}, X_{j}\right], \quad j \in \mathcal{I}_{\mathcal{U} \mathcal{K}}^{(i)}
\end{aligned}
$$

and $\forall s \in\left\{1,2, \ldots, m_{i}\right\}, \mathcal{K}_{s}$ is described in (4), then there exists a mode-dependent stabilizing controller of the form in (5) such that the closed-loop system is stochastically stable. Moreover, if the LMIs in (20) have a solution, an admissible controller gain is given by (17).

Proof: First of all, by Theorem 3, we know that system (2) is stochastically stable with partially unknown transition probabilities if the inequality (19) holds. By Schur complement, (19) is equivalent to

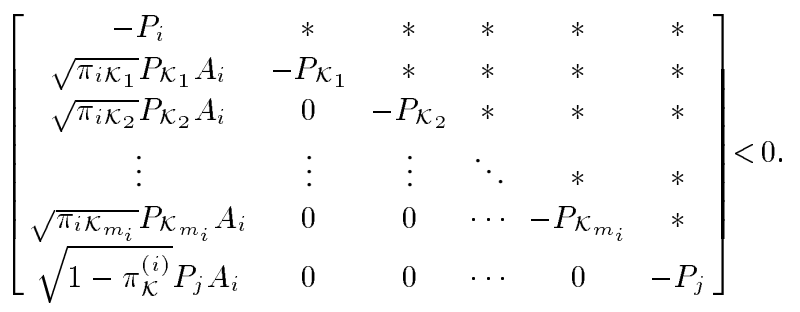

Now, consider the system with the control input (5) and replace $A_{i}$ by $A_{i}+B_{i} K_{i}$ in (23). Setting $X_{i} \triangleq P_{i}^{-1}$, performing a congruence transformation to (23) by $\operatorname{diag}\left[X_{i}, \mathcal{X}_{\mathcal{K}}^{(i)}\right]$ and applying the change of variable $Y_{i} \triangleq K_{i} X_{i}$, we can readily obtain (20). Therefore, if (20) holds, (19) will be satisfied in Theorem 3, that is, the underlying system is stochastically stable. Meanwhile, due to $Y_{i}=K_{i} X_{i}$, the desired controller gain is given by (17).

Remark 5: In contrast with the continuous-time case, the discretetime case is relatively simpler since all the elements in the TPM are nonnegative and we need not distinguish the cases of diagonal elements known or unknown.

Remark 6: It is noted that an interesting conclusion can be directly drawn from Theorem 1 and Theorem 3. That is, when all the elements in the TRM or TPM are unknown, the underlying systems are subject to switchings without known statistics. This leads to the so-called deterministic switched systems under arbitrary switchings (see [19], [20] for continuous-time and discrete-time case, respectively). We can therefore obtain the necessary and sufficient stability criterion of such switched systems in continuous-time and discrete-time cases, respectively. More specifically, in the discrete-time case, we have the stability condition is $A_{i}^{T} P_{j} A_{i}-P_{i}<0, \forall i \times j \in \mathcal{I} \times \mathcal{I}$, which is reduced from (19) when all the elements in the TPM are unknown. Likewise, for the
TABLE I

CONTROLLERS FOR TRM (25)

\begin{tabular}{|c|c|}
\hline \hline Results & \multicolumn{2}{|c|}{ Controller Gains } \\
\hline & $K_{1}=\left[\begin{array}{ll}-0.47 & -0.53\end{array}\right]$ \\
Theorem 2 & $K_{2}=\left[\begin{array}{ll}-0.17 & 0.64\end{array}\right]$ \\
& $K_{3}=\left[\begin{array}{ll}-0.33 & -0.14\end{array}\right]$ \\
\hline Theorem 2 in [15] & \multicolumn{2}{|c}{ Infeasible } \\
\hline \hline
\end{tabular}

continuous-time case, if all the elements in the TRM are unknown, the conditions leads to (9) only and it reduces to

$$
A_{i}^{T} P_{i}+P_{i} A_{i}+\lambda_{d}^{(i)}\left(P_{i}-P_{j}\right)<0 .
$$

Since $\lambda_{d}^{(i)}$ can be arbitrarily negative, inequality (24) requires $P_{i}=$ $P_{j} \equiv P$ which leads to the condition

$$
A_{i}^{T} P+P A_{i}<0, \quad \forall i \times j \in \mathcal{I} \times \mathcal{I} .
$$

\section{Numerical Examples}

The validity and the reduction of conservatism of the results obtained above are verified by the following numerical examples.

Example 1: Consider MJLS (1) with three operation modes and the following system matrices:

$$
\begin{aligned}
& A_{1}=\left[\begin{array}{cc}
-0.50 & -0.75 \\
1 & 1
\end{array}\right], \quad A_{2}=\left[\begin{array}{cc}
-2.4 & -0.33 \\
1 & -1.4
\end{array}\right], \\
& A_{3}=\left[\begin{array}{cc}
-0.20 & 0.1 \\
1 & -1
\end{array}\right], \quad B_{1}=\left[\begin{array}{l}
5 \\
0
\end{array}\right], \\
& B_{2}=\left[\begin{array}{l}
-2 \\
-1
\end{array}\right], \quad B_{3}=\left[\begin{array}{c}
1 \\
-2
\end{array}\right] .
\end{aligned}
$$

Assume the TRM is given by

$T M R=$\begin{tabular}{|c||c|c|c|}
\hline Mode & 1 & 3 & 4 \\
\hline \hline 1 & -1.3 & $\hat{\lambda}_{12}$ & $\hat{\lambda}_{13}$ \\
2 & 0.7 & -1.2 & 0.5 \\
3 & $\hat{\lambda}_{31}$ & $\hat{\lambda}_{32}$ & -0.5 \\
\hline
\end{tabular}

where $\hat{\lambda}_{i j}, \forall i \times j \in \mathcal{I} \times \mathcal{I}_{\mathcal{U} \mathcal{K}}^{(i)}$ denote the unknown elements.

The purpose of this example is to verify the reduced conservatism of the obtained results in the continuous-time case. First, one can check that the open loop system is unstable by both Theorem 1 in the technical note and Theorem 1 in [15]. Then, based on Theorem 2 in the technical note, we obtain the controller gains for the system as shown in Table I. However, it is verified that the stabilization criterion developed previously cannot yield a feasible solution of the controller, which shows that the developed approach in the technical note is less conservative.

Notice that in Example 1, all the diagonal elements of $T R M$ (25) are known. Now we further provide another example with unknown diagonal elements in the TRM to illustrate the dependency of controller design on the lower bound $\lambda_{d}^{(i)}$ of the corresponding unknown diagonal element.

Example 2: Consider MJLS (1) with four operation modes and the following system matrices:

$$
\begin{aligned}
& A_{1}=\left[\begin{array}{cc}
-15 & -7.5 \\
10 & 10
\end{array}\right], \quad A_{2}=\left[\begin{array}{cc}
2.4 & -3.3 \\
10 & 14
\end{array}\right], \\
& A_{3}=\left[\begin{array}{cc}
-2 & 1 \\
10 & 10
\end{array}\right], \quad A_{4}=\left[\begin{array}{cc}
10 & -2.3 \\
10 & -11
\end{array}\right], \\
& B_{1}=\left[\begin{array}{l}
1 \\
0
\end{array}\right], \quad B_{2}=\left[\begin{array}{c}
0 \\
-1
\end{array}\right], \quad B_{3}=\left[\begin{array}{c}
1 \\
-2
\end{array}\right], \quad B_{4}=\left[\begin{array}{c}
-1 \\
1
\end{array}\right] .
\end{aligned}
$$


TABLE II

CONTROLLERS FOR TRM (26)

\begin{tabular}{|c|c|}
\hline \hline Results & Solutions of controller gains \\
\hline \hline & $K_{1}=\left[\begin{array}{ll}-60.69 & -119.67\end{array}\right]$, \\
Theorem 2 $\left(\lambda_{d}^{(2)}=-1\right)$ & $K_{2}=\left[\begin{array}{ll}-1.02 & 0.07\end{array}\right] \times 10^{3}$ \\
& $K_{3}=\left[\begin{array}{ll}96.36 & 81.97\end{array}\right]$, \\
& $K_{4}=\left[\begin{array}{ll}72.16 & 25.86\end{array}\right]$ \\
\hline & $K_{1}=\left[\begin{array}{ll}-132.19 & -219.41\end{array}\right]$, \\
Theorem 2 $\left(\lambda_{d}^{(2)}=-1.5\right)$ & $K_{2}=\left[\begin{array}{ll}-1.78 & 0.13\end{array}\right] \times 10^{3}$ \\
& $K_{3}=\left[\begin{array}{ll}169.73 & 145.87\end{array}\right]$, \\
& $K_{4}=\left[\begin{array}{ll}115.47 & 36.99\end{array}\right]$ \\
\hline Theorem 2 $\left(\lambda_{d}^{(2)}=-2.5\right)$ & Infeasible \\
\hline Theorem 2 in [15] & Infeasible \\
\hline \hline
\end{tabular}

The TRM is given by

\begin{tabular}{|c||c|c|c|c|}
\hline Mode & 1 & 2 & 3 & 4 \\
\hline \hline 1 & -1.3 & 0.2 & $\hat{\lambda}_{13}$ & $\hat{\lambda}_{14}$ \\
\hline \hline 2 & $\hat{\lambda}_{21}$ & $\hat{\lambda}_{22}$ & 0.5 & 0.5 \\
\hline 3 & 0.1 & $\hat{\lambda}_{32}$ & -2.5 & $\hat{\lambda}_{34}$ \\
\hline 4 & 0.4 & 0.2 & 0.6 & -1.2 \\
\hline
\end{tabular}

In the 2nd row of $T R M(26)$, the diagonal element $\hat{\lambda}_{22}$ is unknown, we assign its lower bound $\lambda_{d}^{(2)}$ a priori with different values. It can be checked that the open-loop system is unstable based on Theorem 1 in [15], or Theorem 1 in this technical note for any $\lambda_{d}^{(2)} \in(-\infty,-1]$. Then, by Theorem 2 in [15] and Theorem 2 in the technical note with different $\lambda_{d}^{(2)}$, we obtain the controller gains as shown in Table II.

It is seen from Table II that the obtained controller gains are dependent on $\lambda_{d}^{(2)}$. By applying the bisection method with the conditions in Theorem 2, one can further obtain the minimal value of $\lambda_{d}^{(2)}$, below which the stabilizing controller will not exist (here we get $\underline{\lambda}_{d}^{(2)}=-2.2758$ by some standard numerical software). It is also worth mentioning here that, for some systems, one may obtain that the controller solution is independent on the bound of diagonal elements, as the system in Example 1 of [15] shows that the controller exists despite that $\hat{\lambda}_{22}$ is unknown and has no given lower bound.

Example 3: Consider MJLS (2) with four operation modes and the following system matrices:

$$
\begin{aligned}
A_{1} & =\left[\begin{array}{cc}
1 & -1.25 \\
2.5 & -2.5
\end{array}\right], \quad A_{2}=\left[\begin{array}{cc}
0.25 & -0.83 \\
2.5 & -3.5
\end{array}\right], \\
A_{3} & =\left[\begin{array}{cc}
0.5 & -0.25 \\
2.5 & -3.0
\end{array}\right], \quad A_{4}=\left[\begin{array}{ll}
1.5 & -0.56 \\
2.5 & -2.75
\end{array}\right], \\
B_{1} & =\left[\begin{array}{l}
2 \\
1
\end{array}\right], \quad B_{2}=\left[\begin{array}{c}
1 \\
-1
\end{array}\right], \quad B_{3}=\left[\begin{array}{l}
1 \\
1
\end{array}\right], \quad B_{4}=\left[\begin{array}{l}
0.8 \\
-1
\end{array}\right] .
\end{aligned}
$$

Moreover, the TPM is given by

\begin{tabular}{|c||c|c|c|c|}
\hline Mode & 1 & 4 & 3 & 4 \\
\hline \hline 1 & 0.3 & 0.2 & 0.1 & 0.4 \\
\hline \hline 2 & $\hat{\pi}_{21}$ & 0.2 & 0.3 & $\hat{\pi}_{24}$ \\
\hline 3 & $\hat{\pi}_{31}$ & $\hat{\pi}_{32}$ & -0.5 & 0.5 \\
\hline 4 & 0.2 & 0.2 & 0.1 & 0.5 \\
\hline
\end{tabular}

TABLE III

CONTROLLERS FOR TPM (27)

\begin{tabular}{|c|c|}
\hline \hline Results & Controller Gains \\
\hline \multirow{3}{*}{ Theorem 4 } & $K_{1}=\left[\begin{array}{ll}1.87 & -1.60\end{array}\right]$, \\
& $K_{2}=\left[\begin{array}{ll}1.28 & -1.56\end{array}\right]$ \\
& $K_{3}=\left[\begin{array}{ll}-5.42 & 7.02\end{array}\right]$, \\
& $K_{4}=\left[\begin{array}{ll}0.76 & -1.37\end{array}\right]$ \\
\hline Theorem 4 in [15] & Infeasible \\
\hline
\end{tabular}

The comparison of Theorem 4 in the technical note with Theorem 4 in [15] is summarized in Table III, where the reduction of conservatism of the new criterion is demonstrated.

\section{CONCLUSION}

In this technical note, we have revisited the analysis and synthesis problems of Markov jump linear system with incomplete transition descriptions. Necessary and sufficient criteria are obtained for MJLSs in both continuous-time domain and discrete-time domain by fully exploiting the properties of the transition rates matrix and the transition probabilities matrix. The conservatism of the approach developed previously, which only leads to sufficient conditions for the system, is reduced by the newly developed approach. Numerical examples have verified the theoretical results given in the technical note. It is expected that the approach can be further used for other analysis and synthesis issues such as $H_{\infty}$ analysis, $H_{\infty}$ synthesis and other applications such as Markov jumping neural networks, e.g., [21] with incomplete transition descriptions therein.

\section{ACKNOWLEDGMENT}

The authors would like to thank the associate editor and the reviewers for their helpful comments and suggestions which have helped improve the presentation of the technical note.

\section{REFERENCES}

[1] E. K. Boukas, Stochastic Switching Systems: Analysis and Design. Berlin, Germany: Birkhauser, 2005.

[2] O. L. V. Costa, M. D. Fragoso, and R. P. Marques, Discrete-Time Markovian Jump Linear Systems. $\quad$ London, U.K.: Springer-Verlag, 2005.

[3] Y. G. Fang and K. A. Loparo, "Stabilization of continuous-timie jump linear systems," IEEE Trans. Autom. Control, vol. 47, no. 10, pp. 1590-1603, Oct. 2002

[4] X. Feng, K. A. Loparo, Y. Ji, and H. J. Chizeck, "Stochastic stability properties of jump linear systems," IEEE Trans. Autom. Control, vol. 37, no. 1, pp. 38-52, Jan. 1992.

[5] G. Nakura, "Stochastic optimal tracking with preview by state feedback for linear discrete-time Markovian jump systems," Int. J. Innovative Comp, Inform. Control, vol. 6, no. 1, pp. 15-27, 2010.

[6] P. Shi, E. K. Boukas, and R. K. Agarwal, "Control of Markovian jump discrete-time systems with norm bounded uncertainty and unknown delay," IEEE Trans. Autom. Control, vol. 44, no. 11, pp. 2139-2144, Nov. 1999.

[7] P. Shi, E. K. Boukas, and R. K. Agarwal, "Kalman filtering for continuous-time uncertain systems with Markovian jumping parameters," IEEE Trans. Autom. Control, vol. 44, no. 8, pp. 1592-1597, Aug. 1999.

[8] Z. Wang, Y. Liu, L. Yu, and X. Liu, "Exponential stability of delayed recurrent neural networks with Markovian jumping parameters," Phys. Lett. A, vol. 356, no. 4, pp. 346-352, 2006.

[9] Z. Wang, H. Qiao, and K. Burnham, "On stabilization of bilinear uncertain time-delay stochastic systems with Markovian jumping parameters," IEEE Trans. Autom. Control, vol. 47, no. 4, pp. 640-646, Apr. 2002 . 
[10] S. Xu and T. Chen, "Robust $H_{\infty}$ control for uncertain discrete-time stochastic bilinear systems with Markovian switching," Int. J. Robust Nonlin. Control, vol. 15, no. 5, pp. 201-217, 2005.

[11] Q. Ding and M. Zhong, "On designing $H_{\infty}$ fault detection filter for Markovian jump linear systems with polytopic uncertainties," Int. J. Innovative Comp. Inform. Control, vol. 6, no. 3, pp. 995-1004, 2010.

[12] B. Bercu, F. Dufour, and G. Yin, "Almost sure stabilization for feedback controls of regime-switching linear systems with a hidden Markov chain," IEEE Trans. Autom. Control, vol. 54, no. 9, pp. 2114-2125, Sep. 2009.

[13] C. Schwartz and A. H. Haddad, "Control of jump linear systems having semi-Markov sojourn times," in Proc. IEEE 42nd Conf. Decision Control, Mad, HI, 2003, pp. 2804-2805.

[14] L. X. Zhang, " $H_{\infty}$ estimation for discrete-time piecewise homogeneous Markov jump linear systems," Automatica, vol. 45, no. 11, pp. 2114-2125, 2009.

[15] L. X. Zhang and E. K. Boukas, "Stability and stabilization of Markovian jump linear systems with partly unknown transition probability," Automatica, vol. 45, no. 2, pp. 463-468, 2009.

[16] L. X. Zhang and E. K. Boukas, " $H_{\infty}$ control for discrete-time Markovian jump linear systems with partly unknown transition probabilities," Int. J. Robust Nonlin. Control, vol. 19, no. 5, pp. 868-883, 2009.

[17] L. X. Zhang and E. K. Boukas, " $H_{\infty}$ control of a class of extended Markov jump linear systems," IET Control Theory Appl., vol. 3, no. 7, pp. 834-842, 2009.

[18] L. X. Zhang, E. K. Boukas, and J. Lam, "Analysis and synthesis of Markov jump linear systems with time-varying delays and partially known transition probabilities," IEEE Trans. Autom. Control, vol. 53, no. 10, pp. 2458-2464, Sep. 2008.

[19] J. Daafouz, P. Riedinger, and C. Iung, "Stability analysis and control synthesis for switched systems: A switched Lyapunov function approach," IEEE Trans. Autom. Control, vol. 47, no. 11, pp. 1883-1887, Nov. 2002.

[20] D. Liberzon, Switching in Systems and Control. Berlin, Germany: Birkhauser, 2003.

[21] Y. Liu, Z. Wang, J. Liang, and X. Liu, "Stability and synchronization of discrete-time Markovian jumping neural networks with mixed modedependent time-delays," IEEE Trans. Neural Networks, vol. 20, no. 7, pp. 1102-1116, Jul. 2009.

\section{Topological Obstructions to Submanifold Stabilization}

\author{
Abdol-Reza Mansouri, Member, IEEE
}

Abstract-We consider the problem of local asymptotic feedback stabilization-via a continuously differentiable feedback law-of a control system $\dot{\mathrm{x}}=f(\mathrm{x}, \mathrm{u})$ defined in Euclidean space $\mathbb{R}^{n}$ (with $f$ being continuously differentiable) to a compact, connected, oriented $m$-dimensional submanifold $M$ of $\mathbb{R}^{n}$ with codimension strictly larger than one. We obtain necessary conditions on the topology of $M$ for such a stabilizing feedback law to exist. This extends the work done in [6], where only the codimension one case was treated. We also briefly discuss the case where the control is only assumed continuous.

Index Terms-Euler-Poincare characteristic, homology groups, submanifold stabilization.

\section{INTRODUCTION}

Consider the following modification of Brockett's non-holonomic integrator [1], introduced in [6]: In $\mathbb{R}^{3}$ (with canonical coordinate functions $x, y, z)$, we define

$$
\text { (I) }\left\{\begin{array}{l}
\dot{x}=u, \\
\dot{y}=v, \\
\dot{z}=(y u-x v) e^{z}
\end{array}\right.
$$

where $u, v$ are the control functions. The control function $f$ is given $\begin{aligned} & \text { here, with } \mathbf{x}=\left(\begin{array}{l}x \\ y \\ z\end{array}\right) \text { and } \mathbf{u}=\left(\begin{array}{l}u \\ v\end{array}\right) \text {, by } \\ &(\mathbf{x}, \mathbf{u}) \mapsto f((\mathbf{x}, \mathbf{u}))=\left(\begin{array}{c}u \\ v \\ (y u-x v) e^{z}\end{array}\right)\end{aligned}$

and is continuously differentiable. It is clear that $f$ is not onto any neighborhood of the origin in $\mathbb{R}^{3}$; indeed, no point on the $z$-axis of $\mathbb{R}^{3}$ other than the origin is in the range of $f$. It follows [1] that there exists no continuously differentiable feedback law that can stabilize this system to the origin. Consider now the problem of asymptotically stabilizing this control system to a submanifold of $\mathbb{R}^{3}$ homeomorphic to the unit sphere $S^{2}$ of $\mathbb{R}^{3}$; defining

$$
\Sigma_{f, \infty}=\left\{(\mathbf{x}, \mathbf{u}) \in \mathbb{R}^{3} \times \mathbb{R}^{2} \mid f(\mathbf{x}, \mathbf{u}) \neq 0\right\}
$$

we easily have that $\Sigma_{f, \infty}=\mathbb{R}^{3} \times\left(\mathbb{R}^{2} \backslash\{0\}\right)$; hence, we obtain $H_{2}\left(\Sigma_{f, \infty} ; \mathbb{Z}\right) \simeq H_{2}\left(\mathbb{R}^{2} \backslash\{0\} ; \mathbb{Z}\right)=0$, where $H_{k}(\cdot ; \mathbb{Z})$ denotes the $k^{\text {th }}$ singular homology group with coefficients in $\mathbb{Z}$, and " $\simeq$ " denotes a group isomorphism (see e.g., Chapter 4 of [7]). On the other hand, the Euler-Poincaré characteristic $\chi\left(S^{2}\right)$ of $S^{2}$ is non-zero (see Chapter 4 of [7]). It follows therefore from Theorem 4 of [6] that there exists no continuously differentiable feedback law stabilizing the above control system to $S^{2}$. Consider now the problem of asymptotically stabilizing this control system to the unit circle in the $x y$-plane, defined by $S^{2} \cap\{z=0\}$. As noted in [6], this stabilization is achievable, and

Manuscript received January 28, 2009; revised September 09, 2009. First published April 01, 2010; current version published July 08, 2010. This work was supported in part by the Natural Sciences and Engineering Research Council of Canada. Recommended by Associate Editor D. Liberzon.

The author is with the Department of Mathematics and Statistics, Queen's University, Kingston, ON K7L 3N6, Canada (e-mail: mansouri@mast.queensu. ca).

Digital Object Identifier 10.1109/TAC.2010.2046922 